HEP/123-qed

\title{
Influence of the Characteristics of the STM-tip on the Electroluminescence Spectra
}

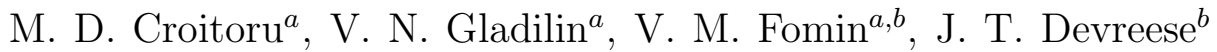 \\ Theoretische Fysica van de Vaste Stoffen (TFVS), Universiteit Antwerpen, Belgium \\ M. Kemerink, P. M. Koenraad, J. H. Wolter \\ COBRA Inter-University Research Institute, \\ Eindhoven University of Technology, The Netherlands
}

(Dated: June 28, 2018)

\begin{abstract}
We analyze the influence of the characteristics of the STM-tip (applied voltage, tip radius) on the electroluminescence spectra from an STM-tip-induced quantum dot taking into account the many-body effects. We find that positions of electroluminescence peaks, attributed to the electronhole recombination in the quantum dot, are very sensitive to the shape and size of the confinement potential as determined by the tip radius and the applied voltage. A critical value of the tip radius is found, at which the luminescence peak positions as a function of the tip radius manifest a transition from decreasing behavior for smaller radii to increasing behavior for larger radii. We find that this critical value of the tip radius is related to the confinement in the lateral and normal direction.
\end{abstract}

PACS numbers: 73.21.La, 78.60.Fi, 73.21.Ac, 78.20.Bh 


\section{INTRODUCTION}

Scanning-tunnelling microscopy techniques have developed into an important tool for studying semiconductor nanostructures in recent years. The scanning-tunnelling microscope (STM) covers a wide field of applications. An example of such an application is the socalled tip-induced quantum dot, a special and unique type of quantum dot that can only be studied using a STM [1, 2, 3, 4]. When a negative bias is applied between the metallic STM-tip and the semiconductor sample, the electric field extends into the structure, and a hole accumulation layer is formed at the surface under the tip. Such an accumulation layer effectively screens the electric field. For sufficiently small tip apex radii, quantization occurs both in the radial direction and along the axis normal to the surface of the structure. It should be noted that the tip-induced band bending confines only a single type of carriers, holes in our case. The electrons are repelled from the region under the tip. By putting a barrier just below the surface, injected electrons are confined to the surface layer and radiative recombination with holes in the dot becomes possible.

In this paper we analyze the influence of the characteristics of the confinement potential, determined by the STM-tip radius and the applied voltage, on the electroluminescence spectra of a tip-induced quantum dot in the GaAs layer of the GaAs $/ \mathrm{Al}_{0.25} \mathrm{Ga}_{0.75} \mathrm{As} / \mathrm{In}_{0.25} \mathrm{Ga}_{0.75}$ As quantum well structure [4].

\section{MODEL}

In our model we consider a multilayer structure sketched in Fig. 1. The shape of the STM tip is modelled by a truncated cone because, when the tip made of a soft metal (like Pt in our case) is pressed to the semiconductor layer, it becomes flat. The STM-tip and the GaAs layer (cap layer) are separated from each other by an insulator layer of width $l_{1}$. The holes and electrons are confined in the cap layer of width $l_{2}$. The width of the $\mathrm{Al}_{0.25} \mathrm{Ga}_{0.75}$ As barrier is $l_{3}$. The $\mathrm{Al}_{0.25} \mathrm{Ga}_{0.75}$ As layer borders on the $\operatorname{In}_{0.25} \mathrm{Ga}_{0.75}$ As quantum well. The contact area of the STM-tip with the structure is a circle of radius $R_{\text {tip. }}$. Owing to the axial symmetry of the system, cylindrical coordinates $r, \varphi, z$ are used. The $z$-axis is directed along the symmetry axis of the tip, which is chosen parallel to the normal to the surface of the structure. While the hole motion is confined in all directions by the potential 
well, the electron motion is confined only along the $z$-axis.

In order to find energy spectra of holes, trapped in the quantum dot, we solve selfconsistently the Poisson equation

$$
\frac{1}{r} \frac{\partial}{\partial r}\left(r \frac{\partial V(r, z)}{\partial r}\right)+\frac{\partial^{2} V(r, z)}{\partial z^{2}}=-\frac{\rho_{h}(r, z)}{\varepsilon_{0} \varepsilon_{i}}
$$

where $\rho_{h}(r, z) \equiv e n_{h}(r, z)$ is the hole charge density and $\varepsilon_{i}$ is the dielectric constant of the material, together with the Schrödinger equation governing the hole motion:

$$
\begin{gathered}
-\frac{\hbar^{2}}{2}\left(\frac{1}{m_{j}^{\|}(z)} \frac{1}{r} \frac{\partial}{\partial r}\left(r \frac{\partial}{\partial r}\right)+\frac{1}{m_{j}^{\|}(z)} \frac{1}{r^{2}} \frac{\partial^{2}}{\partial \varphi^{2}}+\frac{\partial}{\partial z} \frac{1}{m_{j}^{\perp}(z)} \frac{\partial}{\partial z}\right) \Psi_{j, s, n, m}(r, \phi, z) \\
\quad+V(z, r) \Psi_{j, s, n, m}(r, \phi, z)=E_{j, s, n, m} \Psi_{j, s, n, m}(r, \phi, z) \\
m_{j}^{\|, \perp}(z)=\left\{\begin{array}{l}
m_{j}^{\|, \perp[G a A s]}, \text { if } z \in \mathrm{GaAs} \\
m_{j}^{\|, \perp\left[A l_{0.25} G a_{0.75} A s\right]}, \text { if } z \in \mathrm{Al}_{0.25} \mathrm{Ga}_{0.75} \text { As. }
\end{array}\right.
\end{gathered}
$$

Here the index $j$ labels the hole band type: $j=1$ for a hole, which is heavy for the motion along the $z$-axis and light in the plane of the GaAs layer and $j=2$ for a hole, which is light for the motion along the z-axis and heavy in the plane of the GaAs layer. For the case of a quantum well, the light and heavy holes exactly decouple at $\mathbf{k}_{\|}=0$. Since the lateral size of the quantum dot is much larger than the GaAs unit cell, only states around $\mathbf{k}_{\|}=0$ contribute to the wavefunctions in the quantum dot. The effect of coupling between the valence bands is very weak in the close vicinity of the center of the Brillouin zone $\mathbf{k}_{\|}=0$ (see, e.g., Refs. [5, 6]). The index $s$ labels subbands due to the size quantization of the hole degree of freedom along the $z$-axis. The index $n$ is the radial quantum number and $m$ is the angular quantum number of the lateral (in the plane of the GaAs layer) motion of the hole. The kinetic energy of the hole motion along the $z$-axis is larger than the kinetic energy of the lateral motion. (Our calculations show that the charge of holes is concentrated in a relatively thin $(\sim 3 \mathrm{~nm})$ layer near the interface insulator/GaAs. The radial size of the smallest dot considered in this paper is $12 \mathrm{~nm}$.) Hence the adiabatic approach can be used, assuming that the hole degree of freedom along the $z$-axis is "fast" while the lateral one is "slow". Therefore, the hole wave function is represented in a product form: $\Psi_{j, s, n, m}(r, \phi, z)=\Psi_{j, s}^{\perp}(z ; r) \Psi_{j, s, n, m}^{\|}(r) e^{i m \phi}$, where $\Psi_{j, s}^{\perp}(z ; r)$ and $\Psi_{j, s, n, m}^{\|}(r) e^{i m \phi}$ are the wave functions describing the motion along the $z$-axis and the lateral motion, respectively. The 
Schrödinger equation governing $\Psi_{j, s}^{\perp}(z ; r)$ reads

$$
-\frac{\hbar^{2}}{2} \frac{\partial}{\partial z} \frac{1}{m_{j}^{\perp}(z)} \frac{\partial}{\partial z} \Psi_{j, s}^{\perp}(z ; r)+V(r, z) \Psi_{j, s}^{\perp}(z ; r)=E_{j, s}(r) \Psi_{j, s}^{\perp}(z ; r),
$$

where $V(z, r)=e U(z, r)+V_{\mathrm{bar}}^{h}(z)$ with $U(z, r)$, the electrostatic potential. $V_{\mathrm{bar}}^{h}(z)$ represents band offsets for a hole [7].

We solve the Schrödinger equation (3) using the method described in Ref. [8, 9]. It yields the spectrum of the subband energies $E_{j, s}(r)$ and wavefunctions $\Psi_{j, s}^{\perp}(z ; r)$. Each of the energies $E_{j, s}(r)$ determines the top of a hole subband in the structure and plays the role of an adiabatic potential for the lateral motion.

Consequently, the equation, which describes the lateral motion, is as follows:

$$
-\frac{\hbar^{2}}{2 m_{j}^{\|}(z)}\left(\frac{1}{r} \frac{\partial}{\partial r}\left(r \frac{\partial}{\partial r}\right)-\frac{m^{2}}{r^{2}}\right) \Psi_{j, s, n, m}^{\|}(r)+E_{j, s}(r) \Psi_{j, s, n, m}^{\|}(r)=E_{j, s, n, m} \Psi_{j, s, n, m}^{\|}(r) .
$$

After solving this equation as described in Ref. 9], we obtain the energy spectrum of the holes, confined in the STM-tip-induced quantum dot.

Given a particular number of holes in the quantum dot, we find the quasi-Fermi level $E_{F}^{h}$ for holes from the equation:

$$
N_{h}=\sum_{j=1,2} \sum_{s, n, m} \frac{2}{\exp \left(\frac{E_{j, s, n, m}-E_{F}^{h}}{k T}\right)+1} .
$$

Then, within the Hartree approximation scheme, the hole density may be extracted from

$$
n_{h}(r, z)=\sum_{j=1,2} \sum_{s, n, m}\left|\Psi_{j, s, n, m}(r, \phi, z)\right|^{2} \frac{2}{\exp \left(\frac{E_{j, s, n, m}-E_{F}^{h}}{k T}\right)+1} .
$$

This is the basic formula employed to invoke a self-consistent solution to the Schrödinger and Poisson equations.

In order to include in our scheme the exchange effects we use the local density approximation (LDA) as described in [10, 11, 12]

$$
E_{\mathrm{xc}}^{\mathrm{LDA}}[n]=\left.\int \mathrm{d} \mathbf{r} e_{\mathrm{xc}}^{\mathrm{hom}}\left(n_{0}\right)\right|_{n_{0} \longrightarrow n(\mathbf{r})},
$$

where $e_{\mathrm{xc}}^{\mathrm{hom}}$ is the exchange energy of the homogeneous gas per particle.

The exchange energy of a hole gas was evaluated in Ref. [13]. The result for exchange energy per hole is

$$
e_{\mathrm{xc}}^{\mathrm{hom}}(n)=\frac{E_{\mathrm{xc}}^{\mathrm{LDA}}}{N}=-\frac{e^{2}}{4 \pi \varepsilon_{0} \varepsilon} \frac{3}{4 \pi}\left(3 \pi^{2} n\right)^{\frac{1}{3}} \zeta(w),
$$


where the numerical function $\zeta(w)$ is given by

$$
=\frac{w^{4}+3 w^{3}+3 w+1-\frac{3}{4}\left(1-w^{2}\right)^{2} \ln \left|\frac{1+w}{1-w}\right|+\frac{3}{4}\left(1-w^{4}\right) \int_{w}^{1} \frac{\mathrm{d} x}{x} \ln \left|\frac{1+x}{1-x}\right|}{4\left(1+w^{3}\right)^{4 / 3}} .
$$

A parameter $w$ represents the ratio between the light- and heavy-hole Fermi wave vectors:

$$
w \equiv \frac{k_{\mathrm{Fl}}}{k_{\mathrm{Fh}}}=\sqrt{\frac{m_{l}}{m_{h}}} .
$$

In the local density approximation, the exchange potential is related to the exchange energy $e_{\mathrm{xc}}^{\text {hom }}(n)$ by $[12]$

$$
V_{\mathrm{xc}}(\mathbf{r})=\frac{1}{e} \frac{\mathrm{d}}{\mathrm{d} n}\left[n e_{\mathrm{xc}}^{\mathrm{hom}}(n)\right](\mathbf{r}) .
$$

The exchange potential is thus

$$
V_{\mathrm{xc}}(r, z)=-\frac{e}{4 \pi \varepsilon_{0} \varepsilon_{\mathrm{GaAs}}}\left(\frac{3}{\pi} \sum_{j=1,2} \sum_{s, n, m}\left|\Psi_{j, s, n, m}(r, \phi, z)\right|^{2}\right)^{\frac{1}{3}} \zeta(w),
$$

This formula is employed to include exchange corrections within LDA in the self-consistent solution of the Schrödinger and Poisson equations. When $V(r, z)$ is substituted by $V(r, z)+$ $V_{\mathrm{xc}}(r, z)$, we need a straightforward numerical solver as shown in Fig. 2. The iteration procedure, involved to calculate the hole states and the corresponding electrostatic potential is subdivided into four steps. In the first step, the electrostatic potential $V(r, z)$ in the whole structure is calculated in the absence of holes. Then the obtained potential is used as the initial guess in the iteration procedure. In the second step, the Schrödinger equation, describing the hole motion is solved. The obtained wave functions are used to calculate the hole density. In the third step, solving the Poisson equation with the charge density obtained in the previous step yields a new approximation for the electrostatic potential, which is involved in the next iteration. In the fourth step, we use the charge density obtained as a result of the second step to calculate the exchange potential Eq. (12), which is also used in the next iteration. At a given number of holes in the tip-induced quantum dot, the second, the third and the fourth steps are repeated consecutively. This sequence of steps continues till the maximal absolute value of the difference between the values of the electrostatic potential at consecutive iterations becomes less than a certain threshold $(\lesssim 0.1 \mathrm{mV})$, which establishes the measure of the accuracy. When increasing the number of holes in the quantum dot, the 
electrostatic potential, obtained earlier for a smaller number of holes, is used as an initial guess for calculations. This procedure guarantees a continuous link between the solutions obtained at the consecutive numbers of holes.

Given the electrostatic potential formed by the hole charge, we solve the Schrödinger equation for electrons,

$$
\begin{aligned}
-\frac{\hbar^{2}}{2}\left(\frac{1}{m_{e}^{\|}(z)} \frac{1}{r} \frac{\partial}{\partial r}\left(r \frac{\partial}{\partial r}\right)+\right. & \left.\frac{1}{m_{e}^{\|}(z)} \frac{1}{r^{2}} \frac{\partial^{2}}{\partial \varphi^{2}}+\frac{\partial}{\partial z} \frac{1}{m_{e}^{\perp}(z)} \frac{\partial}{\partial z}\right) \Psi_{s_{e}, n_{e}, m_{e}}^{e}(r, \phi, z) \\
& +V^{e}(z, r) \Psi_{s_{e}, n_{e}, m_{e}}^{e}(r, \phi, z)=E_{s_{e}, n_{e}, m_{e}}^{e} \Psi_{s_{e}, n_{e}, m_{e}}^{e}(r, \phi, z)
\end{aligned}
$$

where $V^{e}(z, r)=-e U(z, r)+V_{\mathrm{bar}}^{e}(z), U(z, r)$ is the electrostatic potential, and $V_{\mathrm{bar}}^{e}(z)$ describes band offsets for an electron [7],

$$
m_{e}^{\|, \perp}(z)=\left\{\begin{array}{l}
m_{e}^{\|, \perp[G a A s]}, \text { if } z \in \mathrm{GaAs} \\
m_{e}^{\|, \perp\left[A l_{0.25} G a_{0.75} A s\right]}, \text { if } z \in \mathrm{Al}_{0.25} \mathrm{Ga}_{0.75} \mathrm{As}
\end{array}\right.
$$

The index $s_{e}$ labels subbands due to the size quantization of the electron motion in the direction normal to the surface of the structure. The index $n_{e}$ is the radial quantum number and $m_{e}$ is the angular quantum number of the lateral motion of the electron. The electron wave function is represented in a product form: $\Psi_{s_{e}, n_{e}, m_{e}}^{e}(r, \phi, z)=\Psi_{s_{e}}^{\perp, e}(z ; r) \Psi_{s_{e}, n_{e}, m_{e}}^{\|,, e}(r) e^{i m_{e} \phi}$, where $\Psi_{s_{e}}^{\perp, e}(z ; r)$ and $\Psi_{s_{e}, n_{e}, m_{e}}^{\|, e}(r) e^{i m_{e} \phi}$ are the wave functions describing the motion along the $z$-axis and the lateral motion, respectively.

Our numerical approach to solve the Schrödinger equation, which governs the lateral degree of freedom of the electron, is described in Ref. [9]. The set of energies $E_{s_{e}, n_{e}, m_{e}}^{e}$ and wave functions $\Psi_{s_{e}, n_{e}, m_{e}}^{e}(r, \phi, z)$ together with the set of hole energies $E_{j, s, n, m}$ and wave functions $\Psi_{j, s, n, m}(r, \phi, z)$ are used to describe the electron-hole radiative recombination. The intensity of electroluminescence at a frequency $\Omega$ in the quantum dot created by the STM tip is described by the following expression [5]:

$$
I(\hbar \Omega) \sim \sum_{j, s, n, m} \sum_{s_{e}, n_{e}, m_{e}} f_{h}(j, s, n, m) f_{e}\left(s_{e}, n_{e}, m_{e}\right) P\left(j, s, n, m, s_{e}, n_{e}, m_{e}, \hbar \Omega\right),
$$

where $f_{h}(j, s, n, m)$ and $f_{e}\left(s_{e}, n_{e}, m_{e}\right)$ are, respectively, probabilities of finding holes and electrons in the corresponding quantum states, $P\left(j, s, n, m, s_{e}, n_{e}, m_{e}, \hbar \Omega\right)$ is the probability of the electron-hole recombination. 
We assume that the distribution functions $f_{h}(j, s, n, m)$ is described by the Fermi-Dirac function with the quasi-Fermi level $E_{F}^{h}$ :

$$
f_{h}(j, s, n, m)=\frac{1}{\exp \left(\frac{E_{j, s, n, m}-E_{F}^{h}}{k T}\right)+1} .
$$

Following Ref. [9], we assume that due to the radial accelerating field the non-equilibrium

electron distribution is a strongly decreasing function of the angular quantum number $m_{e}$. Hence, the electron distribution function is approximated as

$$
f_{e}\left(s_{e}, n_{e}, m_{e}\right) \sim \delta_{m_{e} 0}
$$

\section{RESULTS AND DISCUSSION}

The characteristics of the structure under consideration are as follows. The thickness of the vacuum barrier is $l_{1}=0.5 \mathrm{~nm}$, while the thickness of the GaAs and $\mathrm{Al}_{0.25} \mathrm{Ga}_{0.75} \mathrm{As}$ layers are, correspondingly, $l_{2}=17 \mathrm{~nm}$ and $l_{3}=46 \mathrm{~nm}$. The respective dielectric constants are $\varepsilon_{3}=1.0, \varepsilon_{2}=13.2$ and $\varepsilon_{3}=12.2$.

Figure 3 illustrates the position of the calculated peaks in the electroluminescence spectrum as a function of the voltage $V_{\text {tip }}$ applied between the STM-tip and the semiconductor structure at $4.2 \mathrm{~K}$. Clearly, the peak positions as a function of the bias between the sample and the tip reveal a red shift. This is explained as follows. An increase of the voltage shifts the bottom (top) of the dot (antidot) upwards nearly proportional to the applied voltage. Since the number of holes increases with the voltage, the width of the dot in the $z$-direction is reduced due to a stronger screening. Hence, an increase of $V_{\text {tip }}$ leads to a deepening of the potential well for holes and to a rise of the barrier for electrons. This, in its turn, raises the energy of the electron states, which contribute to the recombination, with respect to the bottom of the conduction band in the absence of the applied voltage. The holes experience both the rise of the energy level due to the shift of the bottom of the dot and the lowering of the energy level due to a stronger confinement. The net effect of both trends is an upward shift of the hole energy levels. The upward shift of the hole energy levels with increasing $V_{\text {tip }}$ is larger than that for electrons. Thus, these shifts result in a red shift in the electroluminescence spectrum (see also Ref. [9]). 
In Fig. 4 we present the peak positions for the spectrum shown in the inset of Fig. 3 as a function of the radius of the STM-tip. When the radius of the STM-tip $R_{\text {tip }}>12 \mathrm{~nm}$, one notices that the enlargement of the radius $R_{\text {tip }}$ leads to a rise of the transition energies (blue shift). This is understood on the basis of the fact that an enlargement of the contact area shifts on average the conduction and valence bands upwards, resulting in a rising potential barrier for electrons and a slight deepening of the potential well for holes. Consequently, the recombining electron has to occupy a higher energy level in order to have a considerable overlap integral with a hole wave function. Our calculations show that the upward shift of the hole levels is smaller than the increase of the energy of the recombining electron. So, a change of the contact area influences both hole and electron levels, but due to the weak confinement for holes in the radial direction, the shifts of the electron and the hole levels jointly lead to a blue shift of the transition energy (this behavior of the transition energy as a function of the tip radius has been analyzed in Ref. [9]).

As distinct from that type of behavior, for $R_{\text {tip }}<12 \mathrm{~nm}$ an increase of the contact area between the STM-tip and the semiconductor sample results in a red shift of the transition energy. The explanation of this phenomenon is as follows. When the radius of the STM-tip $R_{\text {tip }}$ is much smaller than the thickness of the GaAs layer, the electrostatic field from the STM-tip hardly extends into this layer, as shown schematically in Fig. 5(a). Hence, the band bending in the GaAs layer is negligible. In this case the energies of the transitions between hole and electron levels are practically equal to those in the case without any external field. An enlargement of the contact area increases the extension of the electrostatic field into the structure, as shown in Fig. 5(b). This effect is similar to that of an increase of the applied voltage. In both cases transition energies become smaller (red shift). However, under the further enlargement of the contact area the electrostatic field within this area extends almost homogeneously over the whole semiconductor layer, see Fig. 5(c). As the luminescence originates in this case from the edge of the STM-tip-induced quantum dot, there occurs a smaller rate of decrease of the transition energies with increasing radius. Finally, when the radius $R_{\text {tip }}>12 \mathrm{~nm}$, the effects giving rise to the blue shift, described in the previous paragraph, prevail.

Thus, we have shown a strong effect of the confinement potential, as determined by the STM-tip radius and the applied voltage, on the electroluminescence peak positions. Namely, while an increase of the voltage on the STM-tip results in a red shift of the electrolumines- 
cence peaks 9], an increase of the contact area between the STM-tip and the semiconductor structure results in a non-monotonous variation of peak positions. Below a certain critical value of the tip radius, the transition energies decrease with increasing radius, while above the critical radius of the tip, the transition energies are an increasing function of radius. These results demonstrate vast possibilities of tuning optical characteristics of the STM-tip induced quantum dot by varying parameters of the STM-tip.

\section{Acknowledgments}

This work has been supported by GOA BOF UA 2000, IUAP, FWO-V projects Nos. G.0274.01, G.0435.03, WOG WO.025.99 (Belgium) and the European Commission GROWTH Programme, NANOMAT project, contract No. G5RD-CT-2001-00545. The research of M.K. has been made possible by a fellowship of the Royal Netherlands Academy of Arts and Sciences.

$\left.{ }^{[a}\right]$ Permanent address: Department of Theoretical Physics, State University of Moldova, str. A. Mateevici 60, MD-2009 Kishinev, Republic of Moldova.

$\left.{ }^{b}\right]$ Also at: Technische Universiteit Eindhoven, P. B. 513, 5600 MB Eindhoven, The Netherlands.

[1] M. Wenderoth, M. A. Rosentreter, K. J. Engel, A. J. Heinrich, M. A. Schneider, and R. G. Ulbrich, Europhys. Lett. 45, 579 (1999).

[2] R. Dombrowski, C. Steinebach, C. Wittneven, M. Morgenstern, and R. Wiesendanger, Phys. Rev. B 59, 8043 (1999).

[3] M. Yamauchi, T. Inoshita, and H. Sakaki, Appl. Phys. Lett. 74, 1582 (1999).

[4] M. Kemerink, K. Sauthoff, P. M. Koenraad, J. W. Gerritsen, H. van Kempen, V. M. Fomin, J. H. Wolter, and J. T. Devreese, Appl. Phys. A. 72, S239 (2001).

[5] G. A. Bastard, Wave Mechanics Applied to Semiconductor Heterostructures (Les Editions de Physique, Paris, 1988).

[6] M. Kemerink, P. M. Koenraad, and J. H. Wolter, Phys. Rev. B 54, 10644 (1996).

[7] P. Harrison, Quantum wells, wires and dots. Theoretical and computational physics (John Willey \& Sons, Chichester, 2000). 
[8] E. P. Pokatilov, V. M. Fomin, S. N. Balaban, V. N. Gladilin, S. N. Klimin, J. T. Devreese, W. Magnus, W. Schoenmaker, N. Collaert, M. Van Rossum, and K. De Meyer, J. Appl. Phys. 85, 6625 (1999).

[9] M. Croitoru, V. N. Gladilin, V. M. Fomin, J. T. Devreese, M. Kemerink, P. M. Koenraad, K. Sauthoff, J. H. Wolter, Phys. Rev. B 68, 195307 (2003).

[10] R. M. Dreizer, E. K. U. Gross, Density Functional Theory. An Approach to the Quantum Mamy-Body Problem (Springer, Berlin, 1990).

[11] R. O. Jones, O. Gunnarsson, Rev. Mod. Phys. 61, 689 (1989).

[12] W. Kohn and L. J. Sham, Phys. Rev. 140, A1133 (1965).

[13] P. A. Bobbert, H. Wieldraaijer, R. van der Weide, M. Kemerink, P. M. Koenraad, J. H. Wolter, Phys. Rev B 56, 3664 (1996). 


\section{FIGURE CAPTIONS}

Fig. 1: Scheme of the tip/insulator/GaAs/ $\mathrm{Al}_{0.25} \mathrm{Ga}_{0.75} \mathrm{As} / \mathrm{In}_{0.25} \mathrm{Ga}_{0.75}$ As quantum well structure. The shown hole density distribution is calculated for the STM-tip voltage $-3.1 \mathrm{~V}$ and the radius of the tip $20 \mathrm{~nm}$.

Fig. 2: Flow diagram of the numerical calculations.

Fig. 3: Positions of peaks in the electroluminescence spectra, calculated for the STM-tipinduced quantum dot at $4.2 \mathrm{~K}, R_{\text {tip }}=12 \mathrm{~nm}$, versus bias $V_{\text {tip }}$. Inset: Typical electroluminescence spectrum calculated for the voltage on the STM-tip $V_{\text {tip }}=-3.1 \mathrm{~V}$ and the STM-tip radius $R_{\text {tip }}=12 \mathrm{~nm}$.

Fig. 4: Positions of the electroluminescence peaks from Fig. 3 as a function of the STM-tip radius at the voltage on the STM-tip $V_{\text {tip }}=-3.1 \mathrm{~V}$.

Fig. 5: Sketch of the electrostatic potential profile for the STM-tip induced quantum dots with small (a), intermediate (b) and large (c) radius. Darker regions correspond to a larger absolute value of the electrostatic potential. 


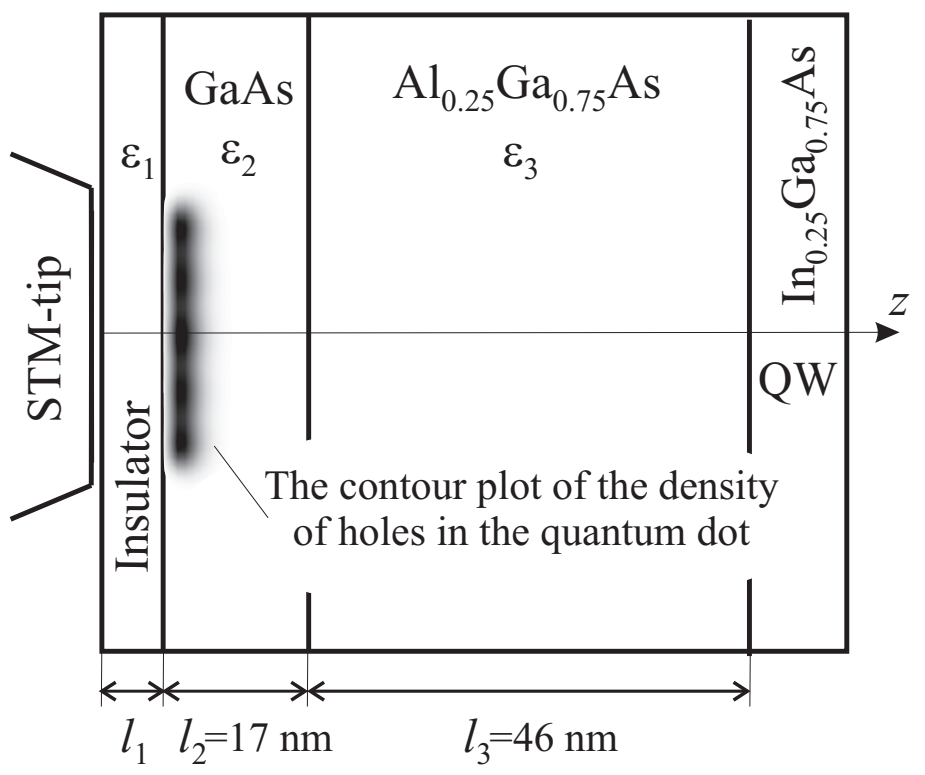

FIG. 1: M. D. Croitoru, V. N. Gladilin, V. M. Fomin, J. T. Devreese, M. Kemerink, P. M. Koenraad, J. H. Wolter 


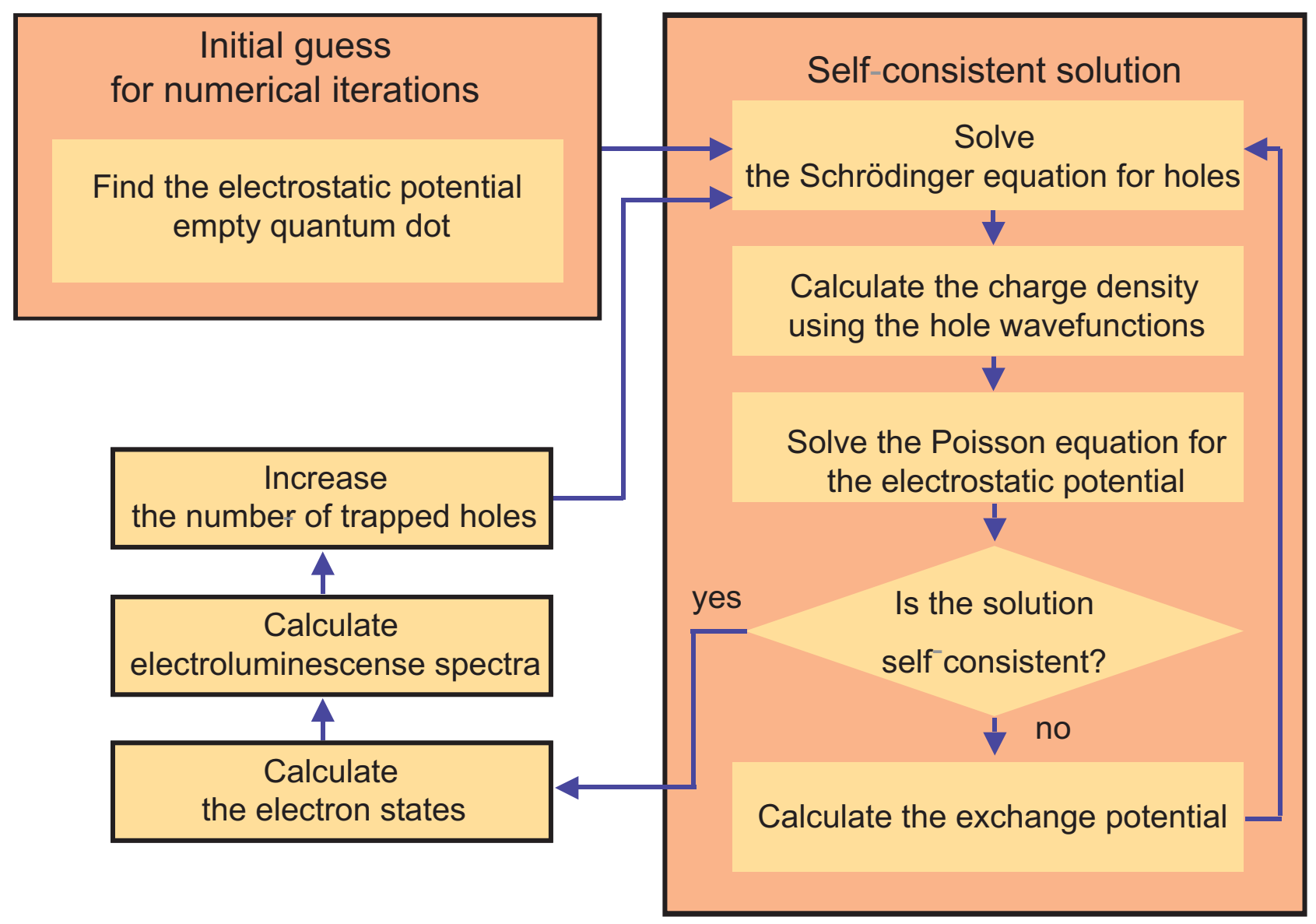

FIG. 2: M. D. Croitoru, V. N. Gladilin, V. M. Fomin, J. T. Devreese, M. Kemerink, P. M. Koenraad, J. H. Wolter 


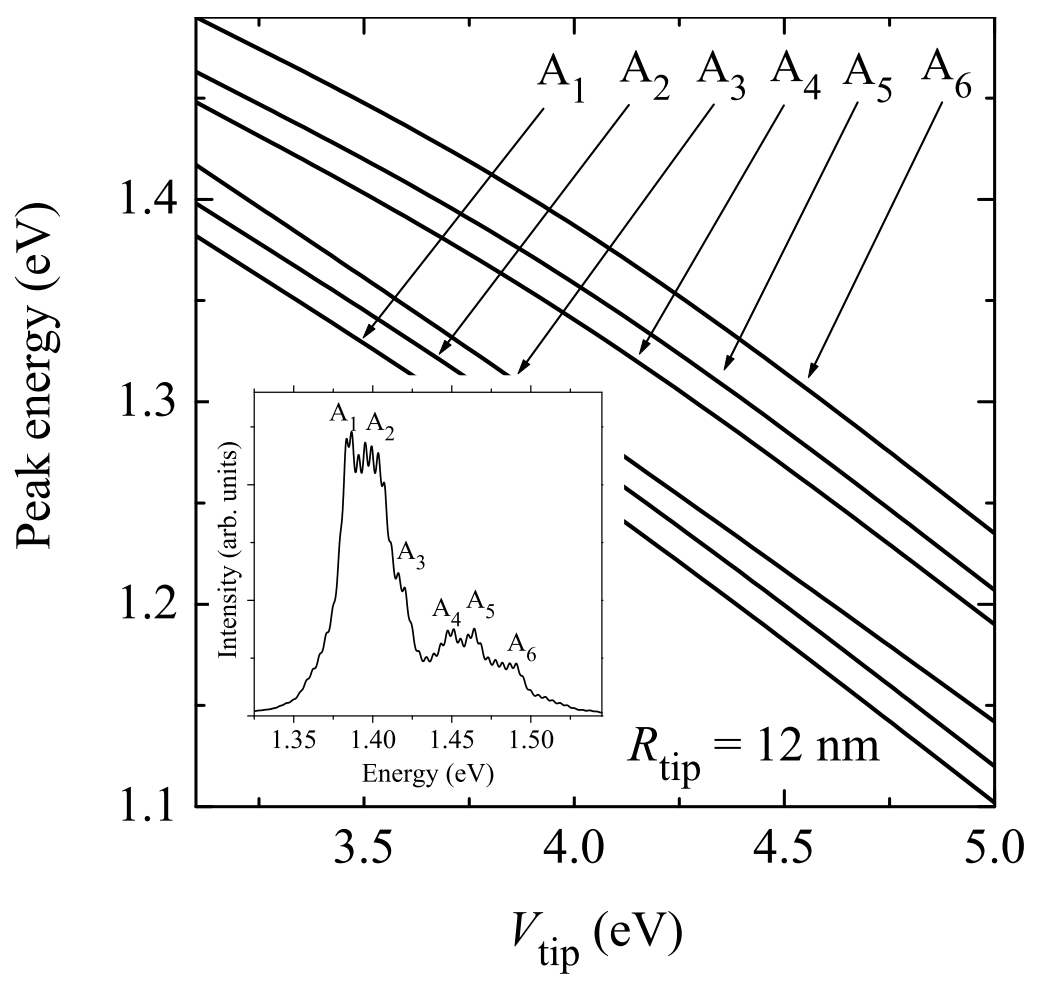

FIG. 3: M. D. Croitoru, V. N. Gladilin, V. M. Fomin, J. T. Devreese, M. Kemerink, P. M. Koenraad, J. H. Wolter 


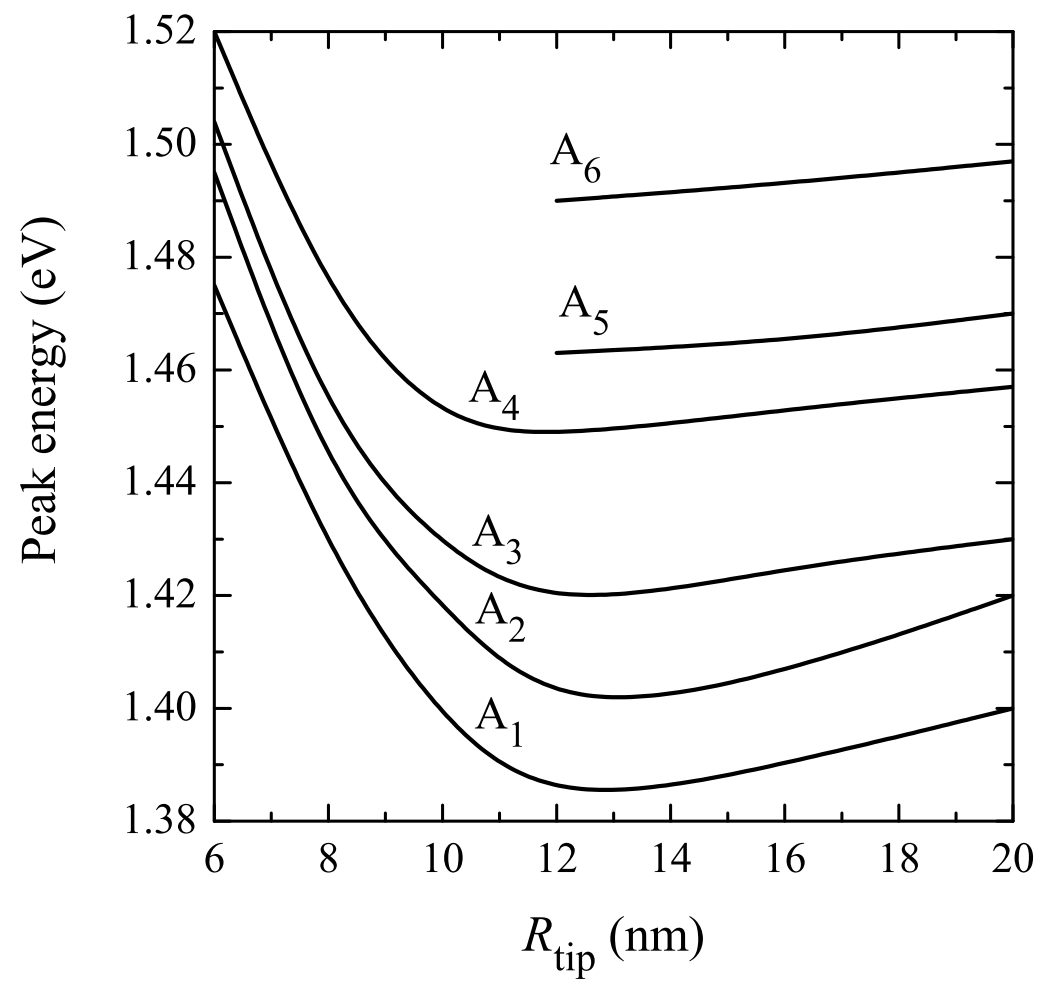

FIG. 4: M. D. Croitoru, V. N. Gladilin, V. M. Fomin, J. T. Devreese, M. Kemerink, P. M. Koenraad, J. H. Wolter

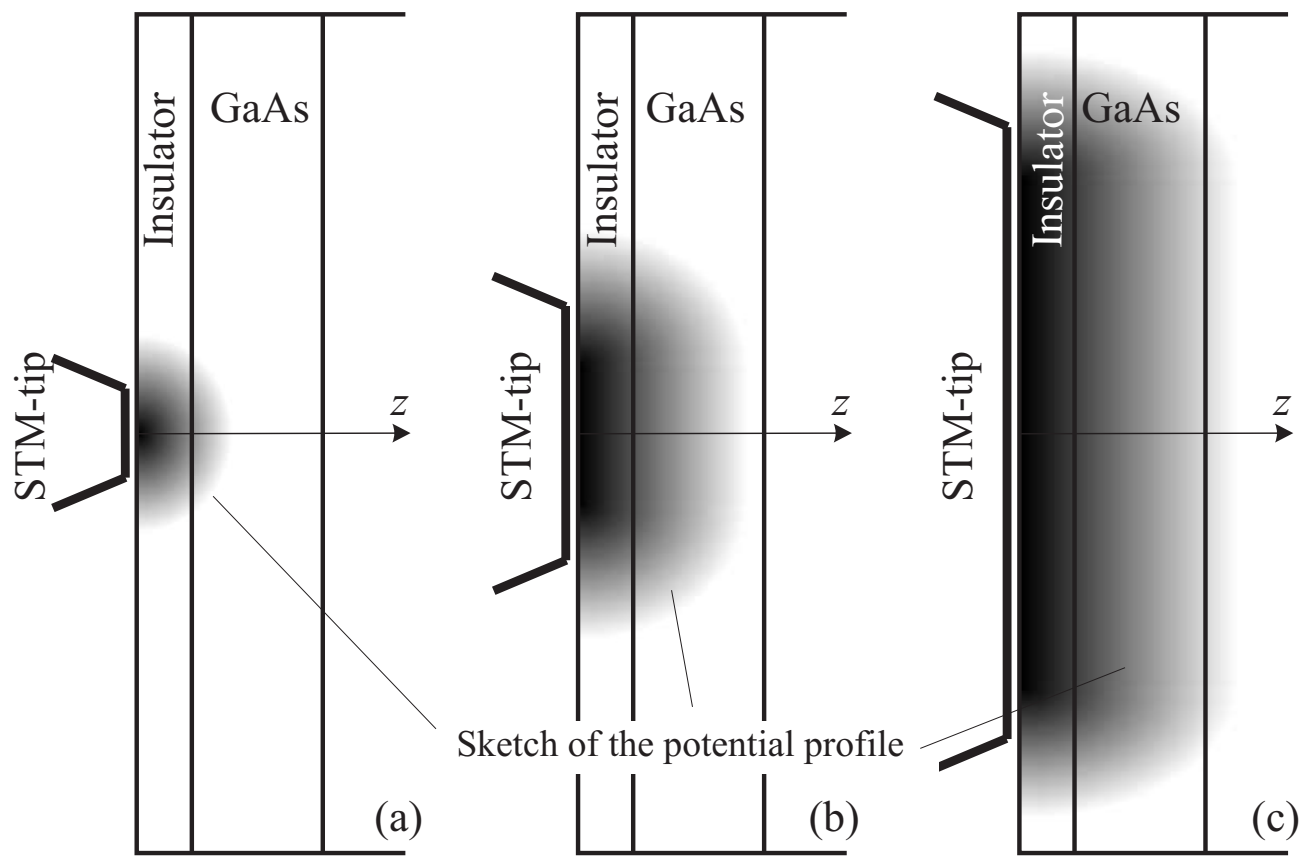

FIG. 5: M. D. Croitoru, V. N. Gladilin, V. M. Fomin, J. T. Devreese, M. Kemerink, P. M. Koenraad, J. H. Wolter 